\title{
Calcification of lower extremity arteries is related to the presence of osteoporosis in postmenopausal women with type 2 diabetes mellitus: a cross-sectional observational study
}

\author{
Xiaojuan $\mathrm{Xu}^{1} \cdot$ Manna Zhang ${ }^{1} \cdot$ Zhaoliang Fei $^{1} \cdot$ Hui Sheng ${ }^{1} \cdot$ Shen $\mathrm{Qu}^{1} \cdot$ Ran Cui $^{1}$ (I)
}

Received: 22 July 2020 / Accepted: 3 December 2020 / Published online: 7 January 2021

(C) The Author(s) 2021

\begin{abstract}
Summary It is unknown whether there is any relationship between extremity arterial macroangiopathy and osteoporosis in type 2 diabetic mellitus (T2DM) patients. We provide evidence to show the association between lower extremity arterial calcification and the presence of osteoporosis in postmenopausal T2DM women, but not in T2DM men of similar age.

Purpose To investigate the relationship between lower extremity arterial calcification and the presence of osteoporosis in type 2 diabetic mellitus (T2DM) patients.

Methods We performed a retrospective cross-sectional study in patients with T2DM. They were assigned into two groups (patients with or without vascular calcification) in both sexes. Clinical characteristics, presence of osteoporosis, and bone metabolic markers were compared. Arterial calcification was determined by ultrasonography examination. Osteoporosis was defined based on the measurements from dual-energy X-ray absorptiometry. The relationship between the lower extremity arterial calcification and the presence of osteoporosis was analyzed. Statistical analysis was performed in SPSS 26.0.

Results A total of 933 T2DM patients ( 535 men $\geq 50$ years old, and 398 postmenopausal women) were identified and analyzed. A significant association between arterial calcification and osteoporosis was only observed in women, with a higher prevalence of osteoporosis observed in women with calcification (40.8\%) than in women without calcification $(26.9 \%)(P=0.004)$. Compared to women without calcification, women with calcification had lower bone mineral densities in the hip $(P<0.001)$ and femoral neck $(P<0.001)$. Ordinal logistic regression analysis showed that women with calcification had a nearly 2 -fold increased risk for osteoporosis, even after adjusting for age, duration of T2DM, body mass index, pulse pressure, clearance of creatinine, glycosylated hemoglobin, and fasting C-peptide. Similar differences were not identified between men with and without calcification. Conclusion Calcification of lower extremity arteries is related with the presence of osteoporosis in postmenopausal T2DM women.
\end{abstract}

Keywords Bone mineral density $\cdot$ Calcification $\cdot$ Diabetes $\cdot$ Lower extremity artery $\cdot$ Osteoporosis

\section{Introduction}

Osteoporosis is a common skeletal condition that affects millions of patients worldwide. In recent decades, etiological

Xiaojuan Xu and Manna Zhang contributed equally to this work.

\section{Ran Cui}

cuiran1172@163.com

Xiaojuan $\mathrm{Xu}$

1833224@tongji.edu.cn

Manna Zhang

mannazhang@126.com

Zhaoliang Fei

feiz@sibcb.ac.cn studies of osteoporosis were mostly focused on the increased osteoclast functions or the decreased osteoblast functions. More recently, vessel abnormalities have become a new research area for osteoporosis. Kusumbe et al. found that type $\mathrm{H}$

Hui Sheng

shenghui@tongji.edu.cn

Shen $\mathrm{Qu}$

qushencn@hotmail.com

Department of Endocrinology and Metabolism, Shanghai Tenth People's Hospital, Tongji University School of Medicine, Shanghai, China 
vessels in a specific murine skeletal system could mediate the growth of the bone vasculature and generate distinct metabolic and molecular microenvironments, as well as maintain perivascular osteoprogenitors and couple angiogenesis to osteogenesis [1]. Molecular frameworks were also identified to couple angiogenesis, angiocrine signals, and osteogenesis [2, 3]. In humans, studies show that type $\mathrm{H}$ vessels are upregulated in aged and osteopenia patients [4]. These findings suggest a link between the vasculature and osteoporosis; however, these human bone samples were collected from patients with hip fractures, which required surgical intervention [4]. More clinical evidence is required to clarify the relationship between vascular abnormalities and osteoporosis.

Type 2 diabetes mellitus (T2DM) is often complicated by vascular diseases. Patients with T2DM had a high risk for peripheral vascular disease, including carotid artery stenosis and lower extremity peripheral artery disease [5]. In our previous studies [6,7], we demonstrated the association between diabetic vascular diseases (microangiopathy and macroangiopathy) and the presence of osteoporosis. Patients with the highest 24-h urine protein levels had the lowest bone mineral density (BMD) in both sexes [6]. Multivariate analysis revealed that diabetic retinopathy was negatively related to BMD in women, but not in men [6]. In addition, we identified a negative correlation between carotid plaque scores and BMD in women with T2DM. A high carotid plaque score with calcification might be a risk factor for osteoporosis [7]. However, research on the relationship between lower extremity arteries and BMD remains limited.

In our previous study, we investigated the relationship between macroangiopathy and BMD in different groups of T2DM patients, including men vs. women, obese vs. nonobese individuals, hypertension vs. non-hypertension, hyperlipidemia vs. non-hyperlipidemia, and calcification vs. noncalcification groups. We found that only sex and calcification were relevant [7]. Therefore, we assumed that the relationship between lower extremity macroangiopathy and BMD could depend on sex and calcification. In the present study, we evaluated the sex and calcification differences in the relationship between lower extremity arteries and the presence of osteoporosis in T2DM patients.

\section{Materials and methods}

\section{Study design and participants}

We conducted a retrospective cross-sectional study. Patients with T2DM who visited the inpatient clinic of the Department of Endocrinology and Metabolism, Shanghai Tenth People's Hospital, Tongji University School of Medicine (China), from July 2018 to February 2020, were reviewed in this study. Study participants were selected based on the inclusion and exclusion criteria. This study was approved by the Ethics
Committee of the hospital. The requirement for informed consent was waived due to the retrospective design of the study.

\section{Inclusion and exclusion criteria}

Inclusion criteria were (1) newly diagnosed T2DM patients or T2DM patients with diabetes-associated complications (e.g., uncontrolled blood glucose, diabetic neuropathy, diabetic nephropathy, and diabetic retinopathy). Diabetes mellitus was diagnosed by either fasting plasma glucose (FPG) $(\geq 7.0 \mathrm{mmol} / \mathrm{l}), 2$ - $\mathrm{h}$ plasma glucose (2-h PG) concentration $(\geq 11.1 \mathrm{mmol} / \mathrm{l})$ after a $75-\mathrm{g}$ oral glucose tolerance test (OGTT) [8], or a history of diabetes. T2DM was diagnosed based on the serum insulin or C-peptide level and diabetic-associated antibodies (glutamic acid decarboxylase antibody, insulin autoantibody, and islet cell antibody) [9]; (2) patients with complete data of color Doppler ultrasonography of lower extremity arteries and dual-energy X-ray absorptiometry (DXA) for BMD. Exclusion criteria were (1) male patients $<50$ years old or premenopausal female patients (these two groups were excluded due to their small sample sizes); (2) patients with severe end-stage renal disease (a history of renal dysfunction or clearance of creatinine less than $30 \mathrm{ml} / \mathrm{min}$ ), liver disease (a history of liver dysfunction or alanine aminotransferase more than $100 \mathrm{U} / \mathrm{L}$ ), or cardiac issues (a history of heart dysfunction or ejection fraction less than 50\%); (3) patients with a history of secondary osteoporosis or those diagnosed after routine examination; (4) patients on medications that could influence bone metabolism, including bisphosphonates, calcitonin, selective estrogen receptor modulators (SERMs), estrogens, or glucocorticoid. For patients with repeat hospital visits, we only analyzed their data collected during the first visit.

Sample size was calculated according to the formula for a cross-sectional study $\left(\mathrm{N}=\mathrm{Z}_{1-\alpha}^{2} * p^{*}(1-p) / d^{2}\right)$. The study required more than 189 men $\left(\mathrm{Z}_{1-\alpha}=1.96, p=0.144, d=0.05\right)$ and 124 women $\left(Z_{1-\alpha}=1.96, p=0.207, d=0.05\right)$.

\section{Demographic variables}

Medical records on age, sex, and T2DM duration were collected. Blood pressure, height, and weight were documented. Pulse pressure (an index of arteriosclerotic stiffness and impaired arterial conduit according to Windkessel physiology) was calculated as the systolic blood pressure minus the diastolic blood pressure. Body mass index (BMI, $\mathrm{kg} / \mathrm{m}^{2}$ ) was also calculated. Information on current medications and menopausal status for women was collected from the electronic and paper medical records.

\section{Laboratory measurements}

Laboratory test results were collected from electronic medical records. The methods of testing were as follows. Fasting blood was collected after overnight fasting. Alanine aminotransferase (ALT), serum creatinine ( $\mathrm{SCr}$ ), and serum calcium and 
phosphorus were analyzed by a routine auto-analyzer (Modular DP analyzer, Roche Diagnostics, Mannheim, Germany). Total cholesterol (TC) and triglycerides (TG) were determined by the enzyme-linked immunosorbent assay (ELISA) method. Clearance of creatinine (CLCR) was calculated by the Cockcroft-Gault formula (men: CLCR $=((140-$ age [years] $) *$ body weight $[\mathrm{kg}]) /(0.818 * \mathrm{SCr}[\mu \mathrm{mol} / 1])$; women: $\mathrm{CLCR}=((140$-age [years] $) *$ body weight $[\mathrm{kg}]) * 0.85) /$ $(0.818 * \mathrm{SCr}[\mu \mathrm{mol} / 1])$. Hemoglobin A1 c (HbA1c) was determined by high-performance liquid chromatography (HPLC) with a coefficient of variation (CV) of $1.11 \%$. Serum fasting C-peptide (CP), osteocalcin, C-terminal telopeptide fragments of type I collagen ( $\beta$-CTX), N-terminal propeptide of type I procollagen (PINP), bone alkaline phosphatase (BAP), parathyroid hormone $(\mathrm{PTH})$, and $25(\mathrm{OH})$ vitamin D were measured using radioimmunoassay (RIA).

\section{Ultrasonography of the lower extremity arteries}

Ultrasonography test results were collected from electronic medical records. The methods of testing were as follows. Lower extremity arteries, including the common femoral artery, superficial femoral artery, popliteal artery, and dorsal artery of the foot, were assessed by high-resolution B-mode and color Doppler/ pulsed-wave Doppler ultrasonography (GE LO5, LOGIQ 5, USA) with a transducer frequency of $7.5 \mathrm{MHz}$. Plaques were identified by the hyperechogenic images inside the artery. Calcification was identified by the bright white echoes protruding into the lumen. The study participants were assigned into four groups based on their calcification status and sex.

\section{Bone mineral density}

Values for the areal BMD $\left(\mathrm{g} / \mathrm{cm}^{2}\right)$ at the lumbar spine (L1-L4), hip, and femoral neck were obtained by DXA on a Hologic QDR $4500 \mathrm{~W}$ densitometer with software version 9.03 (Hologic, USA). All examinations were performed on one device and by one technologist on the same settings following standardized procedures. The BMD devices provided absolute values for the lumbar spine, hip, and femoral neck, along with T-scores or Zscores. The osteoporosis diagnostic criteria [10] from the World Health Organization in postmenopausal women and men aged $\geq$ 50 years were based on the T-score. Normal BMD was defined as a $\mathrm{T}$-score $\geq-1.0$. Osteopenia was defined as a T-score from $>$ -2.5 to $<-1.0$. Osteoporosis was defined as a value for T-score $\leq-2.5 \mathrm{SD}$ in any of the three sites.

\section{Statistical analysis}

Normally distributed continuous data are presented as means \pm SD. Non-normally distributed continuous data are presented as medians and interquartile range (IQR). Categorical data are presented as percentages. To compare the patients with or without vascular calcification in both sexes, independent $t$ test or MannWhitney $U$ test was performed when appropriate. A chi-square test was conducted to compare the difference of osteoporosis between patients with or without calcification. The relationships of lower extremity arteries with prevalence of osteoporosis were further analyzed by ordinal logistic regression analysis. The dependent variables were normal BMD, osteopenia, and osteoporosis. The independent variables that were included in the regression analysis were based on the clinical significance and statistical significance (bivariate analysis with a $P<0.2$ ). Potential confounding factors included age, the duration of T2DM, BMI, pulse pressure, CLCR, glycosylated hemoglobin, and fasting Cpeptide. ORs and corresponding $95 \%$ confidence intervals are reported. Statistical analysis was conducted in SPSS 26.0 (SPSS Inc., Chicago, IL, USA). A two-sided $P<0.05$ was considered statistically significant.

\section{Results}

\section{Clinical characteristics of patients}

A total number of 1,112 T2DM patients (656 men and 456 women) with complete data for color Doppler ultrasonography of lower extremity arteries and DXA for BMD were reviewed. After considering the inclusion and exclusion criteria, 535 men and 398 women were enrolled in our study; these values are larger than the calculated required sample sizes for men and women (Fig. 1).

Clinical characteristics of the study participants stratified by sex and calcification are presented in Table 1. In men, patients with calcification were older than those without calcification. In women, age, duration of T2DM, ALT, SCr, and CLCR were significantly different between those with and without calcification. In both T2DM men and women, there were significant differences in the pulse pressure between those with and without arterial calcification.

\section{Differences in the presence of osteoporosis and bone turnover markers grouped by sex and calcification}

The discrepancies in the presence of osteoporosis and bone turnover markers were different between the calcification and without calcification groups of men and women.

In men, there was no difference in the presence of osteoporosis between the two groups (Table 1). Accordingly, the absolute values (Fig. 2a) and T-scores of BMD (Fig. 2b) at the three sites of lumbar, right hip, and femur neck were not significantly different. Among the bone turnover markers, the level of $25(\mathrm{OH}) \mathrm{VitD}$ was lower in the group with calcification than without calcification (Table 2).

A noticeable difference in the presence of osteoporosis was observed between the two groups of women. The presence of 
osteoporosis in women with calcification $(40.8 \%)$ was significantly higher than that in women without calcification (26.9\%) (Table 1). At the three sites of BMD, the hip and femur neck were lower in the group with calcification than in that without (Fig. 2c). The same trend was discovered for the T-score at the sites of the hip and femur neck (Fig. 2d). However, serum markers of bone resorption and bone formation, including osteocalcin, PINP, $\beta$-CTX, and BAP, as well as calcium and phosphorus metabolism markers, such as serum calcium, phosphorus, $\mathrm{PTH}$, and $25(\mathrm{OH})$ VitD levels, did not show significant differences between the two groups (Table 2).

\section{Ordinal logistic regression analysis between lower extremity arterial calcification and osteoporosis}

In the multivariate analysis, an independent risk factor associated with osteoporosis was vascular calcification in women. Even after adjusting for age, the duration of T2DM, body mass index, pulse pressure, CLCR, glycosylated hemoglobin, and fasting C-peptide in the models, this relationship still existed. In the three models, patients with vascular calcification nearly had double the risk for osteoporosis (model 1: OR 1.92 (1.31, 2.82), $P=0.001$; model 2: OR 1.89 (1.28, 2.78), $P$ $=0.001$; model 3 : OR $1.91(1.29,2.83), P=0.001)$; this relationship was not found in men (Fig. 3).

\section{Discussion}

In this study, we found that lower extremity arteries with calcification doubled the risk for osteoporosis in T2DM women after adjusting for several other risk factors (i.e., age, the duration of T2DM, body mass index, pulse pressure, clearance of creatinine, glycosylated hemoglobin , and fasting C- peptide), not in men. These findings are consistent with the hypothesis that lower extremity arteries are associated with osteoporosis depending on calcification and sex. Studies on the relationship between lower extremity arteries and bone are limited. However, there is some evidence indicating a relationship between atherosclerosis calcification and BMD. For example, Alan et al. found a significant association between cardiovascular disease and low bone mass [11]. BMD is a promising marker for the severity of coronary lesions [12] and coronary artery calcium [13]. Previous studies including ours [7] have suggested that calcification, not plagues, is associated with low BMD and higher risk for osteoporosis. The mechanism was considered to be the effects of chronic inflammation and/or the oxidative stress of calcium metabolism [11-13]. Risk factors, such as aging and decreased levels of sex hormones (especially estrogen), could lead to increased levels of inflammatory and oxidative stress cytokines $[14,15]$. These cytokines, such as tumor necrosis factor- $\alpha$ and interleukin-1 $\beta$, could increase the level of receptor activator of nuclear factor kappa $\beta$ ligand (RANKL), which further activates osteoclasts and results in a shift of calcium from bones to the vessel walls [16]. This shift of calcium might lead to calcification of atherosclerosis and low BMD. Calcification forms specifically in the muscle cells adjacent to atheromas as well as on the surface of atheroma plaques and tissues. However, in this study, differences in the bone metabolic markers were not found to be significant. It is possible that the change in bone turnover markers was blunted in diabetes mellitus [17]. It should be mentioned that medial artery calcification (MAC) is another type of arterial calcification different from atherosclerosis with calcification. MAC, also known as Mönckeberg's arteriosclerosis, is a condition that leads to the stiffening of the elastic layer of the arterial wall. It is also a common feature of conduit artery disease in T2DM [18]. However, in contrast to intimal arterial calcification, MAC

Fig. 1 Flow chart of the study

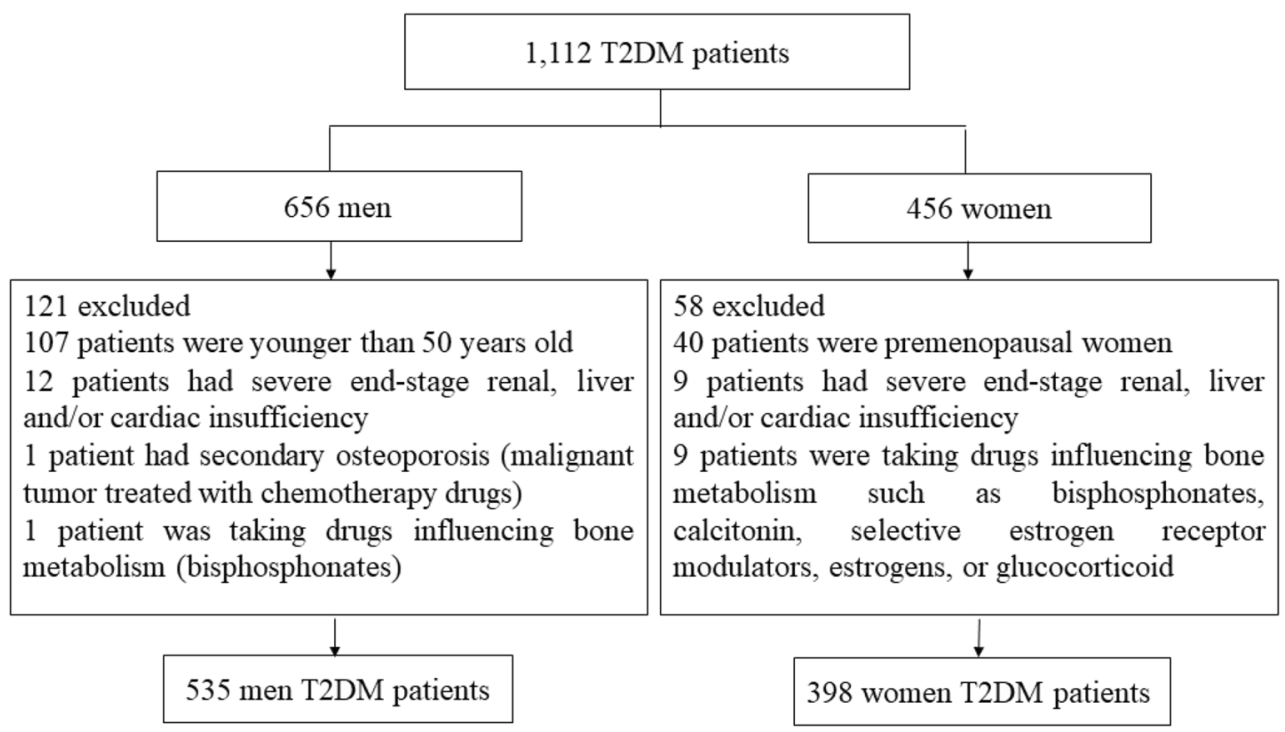




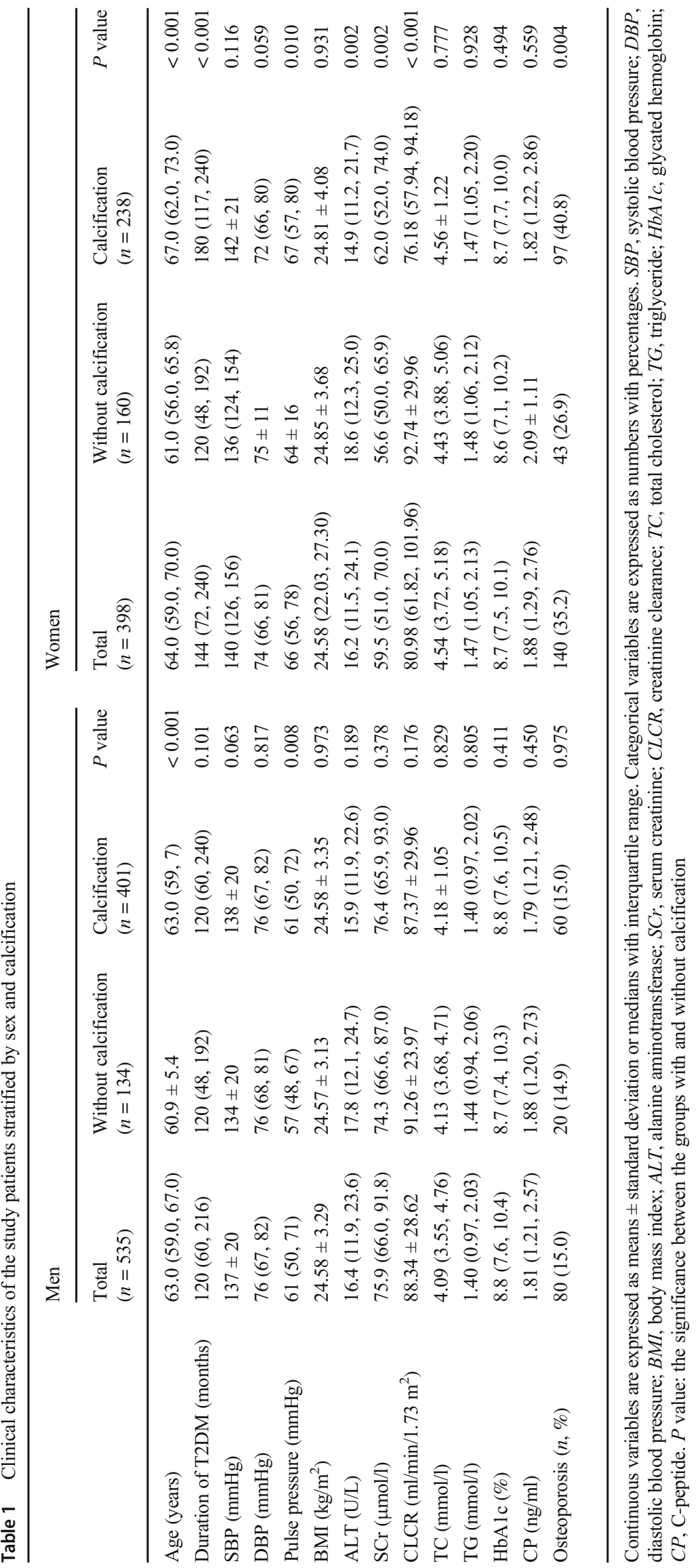




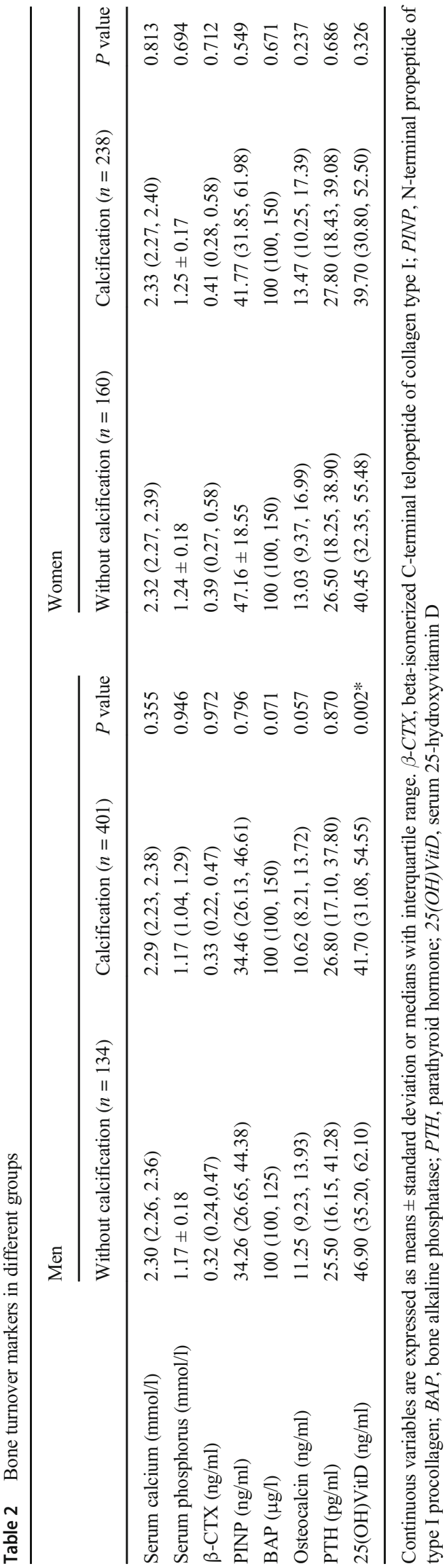

did not obstruct the arterial lumen [19]. In the current study, we did not report MAC in the recruited T2DM patients by ultrasonography. The reason for not reporting MAC is that MAC is better identified by radiogram, whereas atherosclerosis calcification is commonly detected by ultrasonography [20]. In this retrospective study, our patients only received an ultrasonography examination. Moreover, MAC was more frequently observed in patients with diabetic nephropathy, particularly those on hemodialysis [20-22]. In our study, patients with impaired renal function were excluded from the analysis. In the future, we plan to perform prospective studies to independently assess atherosclerosis vs. medial calcification and their relationships with osteoporosis risk.

We found that the correlation between lower extremity arterial calcification and osteoporosis only existed in postmenopausal women, not in men, for unclear reasons. Similar sex differences in association between vascular diseases and BMD have previously been reported in our studies $[6,7]$. Other research has also shown that postmenopausal women with vascular diseases were vulnerable to osteoporosis [23-25]. This might be because postmenopausal women, as compared to men, have a high risk to develop osteoporosis. It is worth mentioning that, in a previous longitudinal study (MrOS), Cummings and colleagues [26] demonstrated high rates of hip bone loss and increased fracture risk in older men with peripheral arterial disease. The participants in the MrOS study were community-dwelling who had a lower prevalence of diabetes mellitus. They were grouped by peripheral artery disease as measured by ankle-brachial index (ABI). We speculate that different study populations, different testing methods, and different groupings led to the results different from ours. Sex differences might be attributable to sex hormones, though this requires further study.

To the best of our knowledge, these data are the first to demonstrate a relationship between lower extremity arterial calcification and the presence of osteoporosis. Interestingly, we found that lower extremity arterial calcification was related with lower BMD of the hip and femur neck. The BMD of the lumbar spine was not different between groups with or without calcification. This is different from the relationship between carotid arterial calcification and BMD, which is related with lower BMD of lumbar spine, hip, and femur neck [7]. The lower extremity arteries include the common femoral artery, superficial femoral artery, popliteal artery, and dorsal artery of the foot. These four arteries provide blood to the hip and femoral neck. The lumbar spine is supplied with blood by the lumbar arteries, which were not examined in this study. The calcification of lumbar arteries was thus not clear. It might be that lower extremity arterial calcification is more closely associated with the BMD of the hip and femur neck. Moreover, the BMD of the lumbar spine might also be related to age, weight, BMI, or estradiol [27, 28]. Nevertheless, we found that the prevalence of osteoporosis was higher in patients with calcification in women. 

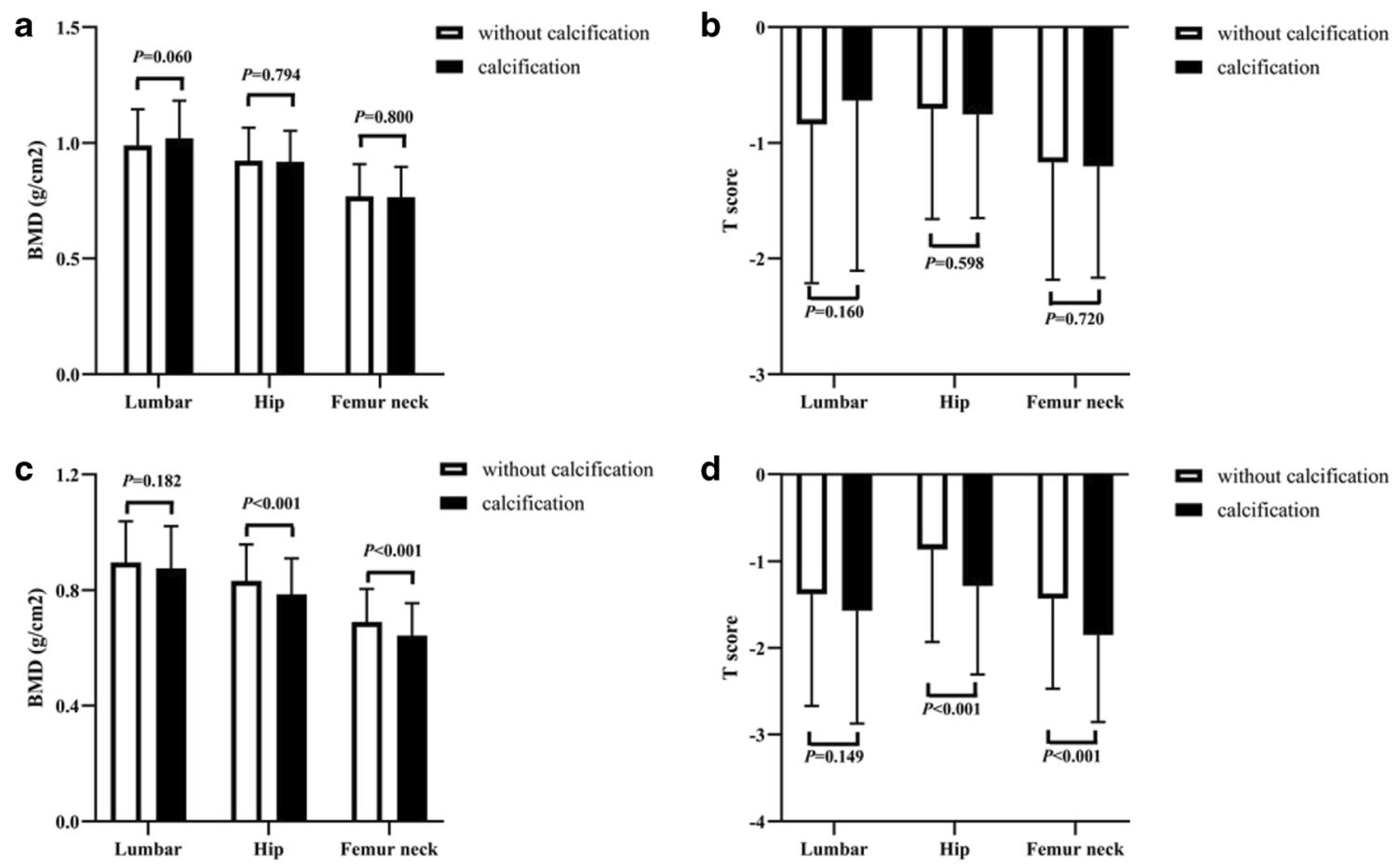

Fig. 2 Difference of BMD by calcification and sex. a BMD value of three sites in men. Lumbar $0.990 \pm 0.155$ vs. $1.020 \pm 0.162, P=0.060$; hip $0.923 \pm 0.144$ vs. $0.919 \pm 0.134, P=0.794$; femur neck $0.769 \pm 0.140$ vs. $0.765 \pm 0.132, P=0.800$. b T-scores of three sites in men. Lumbar $0.840 \pm 1.374$ vs. $-0.636 \pm 1.471, P=0.160$; hip $-0.707 \pm 0.951$ vs. $0.755 \pm 0.894, P=0.598$; femur neck $-1.168 \pm 1.017$ vs. $-1.203 \pm$

Notably, the relationship between vascular disease and abnormal BMD is not the same in microangiopathy and macroangiopathy. In our previous study, the severity of microangiopathy was related to low BMD in women T2DM patients [6]. However, in the current study, a relationship between macroangiopathy without calcification and BMD was not identified. This might indicate that macrovascular disease and microvascular disease affect osteoporosis by different mechanisms. We inferred that macrovasculature results in the infusion of bone; however, microvasculature not only provides nutrients to the bone but also adjusts bone health $[8,29]$.

$0.963, P=0.720$. c BMD value of three sites in women. Lumbar $0.896 \pm$ 0.142 vs. $0.876 \pm 0.145, P=0.182$; hip $0.832 \pm 0.126$ vs. $0.786 \pm 0.125, P$ $<0.001$; femur neck $0.690 \pm 0.115$ vs. $0.643 \pm 0.112, P<0.001$. d Tscore of three sites in women. Lumbar $-1.381 \pm 1.289$ vs. $-1.572 \pm$ $1.302, P=0.149$; hip $-0.886 \pm 1.066$ vs. $-1.285 \pm 1.023, P<0.001$; femur neck $-1.431 \pm 1.042$ vs. $-1.853 \pm 1.003, P<0.001$

Thus, more studies should be performed to investigate the role of vasculopathy in the process of osteoporosis.

In addition to the inherent biases in the retrospective investigation, there are other limitations in the present study. First, there was a lack of data on bone fractures. We will perform additional studies to collect fracture information in future research. Second, cause-and-effect relationships between arterial calcification and osteoporosis could not be determined from the present study due to the cross-sectional study design. Third, since the main complaints of these patients visiting the hospital were for diabetes mellitus instead of osteoporosis,

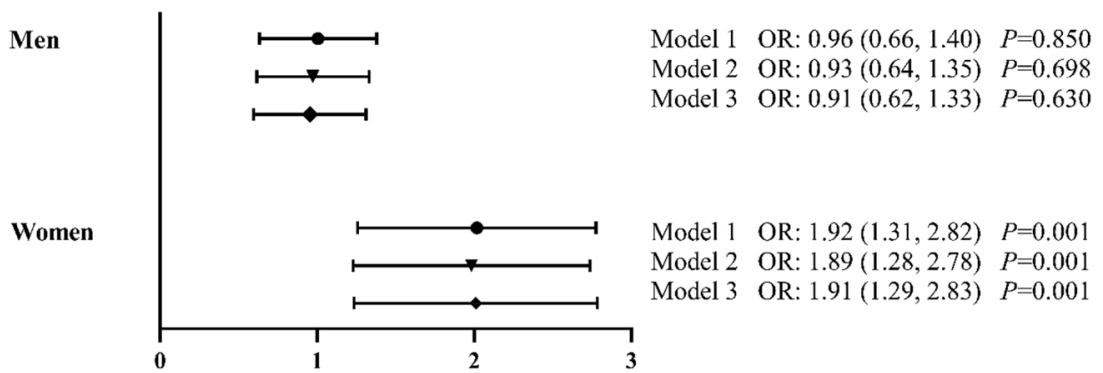

Fig. 3 Ordinal logistic regression analysis of factors associated with osteoporosis. Dependent variables included normal BMD, osteopenia, and osteoporosis. Independent variables included arterial calcification and various potential confounding factors in model 1: age, duration of T2DM, body mass index, and pulse pressure; in model 2: age, duration of

T2DM, body mass index, pulse pressure, and creatinine clearance; and in model 3: age, duration of T2DM, body mass index, pulse pressure, creatinine clearance, glycosylated hemoglobin, and fasting C-peptide. $\mathrm{OR}$, odds ratio 
factors that could potentially affect osteoporosis, such as nutritional status (e.g., calcium and protein intake) and weightbearing physical activity, were not recorded for the analysis. Fourth, granularity of the data on lower extremity arterial calcification, such as details on calcification of each artery, number of vascular beds with calcification, and the extent of calcification, could not be traced in this retrospective observational study. Finally, we performed this study in patients with T2DM, which limited the generalizability of our study results to patients without this disease.

\section{Conclusion}

The present study demonstrated a positive association between calcification of lower extremity arteries and the presence of osteoporosis in postmenopausal women with T2DM. Therefore, patients with calcified lower extremity arteries should be wary of osteoporosis risk. Patients with osteoporosis should also receive examinations on the lower extremity arteries. This relationship was not identified in male patients with or without calcification. In addition, the relationship between macroangiopathy and bone mineral density in T2DM patients depends on vascular calcification and sex, and is different from that found in microangiopathy. Further studies are warranted.

Supplementary Information The online version contains supplementary material available at https://doi.org/10.1007/s00198-020-05775-5.

Acknowledgements This study was supported by the National Natural Science Foundation of China (NSFC 81500650) and the "Climbing" Program of Shanghai Tenth People's Hospital of Tongji University (2018SYPDRC049).

\section{Compliance with ethical standards}

Competing interests The authors declare that they have no competing interests.

Ethics approval and consent to participate This study was approved by the Ethics Committees of Shanghai Tenth People's Hospital, Tongji University School of Medicine, and the requirement of informed consent was waived due to the retrospective design of the study.

Abbreviations T2DM, type 2 diabetes mellitus; BMD, bone mineral density; FPG, fasting plasma glucose; 2-h PG, 2-h plasma glucose; OGTT, oral glucose tolerance test; SERMS, selective estrogen receptor modulators; BMI, $\mathrm{kg} / \mathrm{m}^{2}$, body mass index; ALT, alanine aminotransferase; $\mathrm{SCr}$, serum creatinine; $\mathrm{TC}$, total cholesterol; $\mathrm{TG}$, triglyceride; ELISA, enzyme-linked immunosorbent assay; CLCR, clearance of creatinine; HbA1c, hemoglobin A1c; HPLC, high-performance liquid chromatography; $\mathrm{CV}$, coefficient of variation; $\mathrm{CP}, \mathrm{C}$-peptide; $\beta$-CTX, C-terminal telopeptide fragments of type I collagen; PINP, N-terminal propeptide of type I procollagen; PTH, parathyroid hormone; RIA, radioimmunoassay; DEXA, dual-energy x-ray absorptiometry; SD, standard deviation; IQR, interquartile range; ORs, odds ratios; BAP, bone alkaline phosphatase; RANKL, receptor activator of nuclear factor kappa $\beta$ ligand

Open Access This article is licensed under a Creative Commons Attribution-NonCommercial 4.0 International License, which permits any non-commercial use, sharing, adaptation, distribution and reproduction in any medium or format, as long as you give appropriate credit to the original author(s) and the source, provide a link to the Creative Commons licence, and indicate if changes were made. The images or other third party material in this article are included in the article's Creative Commons licence, unless indicated otherwise in a credit line to the material. If material is not included in the article's Creative Commons licence and your intended use is not permitted by statutory regulation or exceeds the permitted use, you will need to obtain permission directly from the copyright holder. To view a copy of this licence, visit http://creativecommons.org/licenses/by-nc/4.0/.

\section{References}

1. Kusumbe AP, Ramasamy SK, Adams RH (2014) Coupling of angiogenesis and osteogenesis by a specific vessel subtype in bone [J]. Nature. 507(7492):323-328

2. Ramasamy SK, Kusumbe AP, Wang L, Adams RH (2014) Endothelial Notch activity promotes angiogenesis and osteogenesis in bone [J]. Nature. 507(7492):376-380

3. Fu R, Lv WC, Xu Y, Gong MY, Chen XJ, Jiang N, Xu Y, Yao QQ, di L, Lu T, Wang LM, Mo R, Wu ZQ (2020) Endothelial ZEB1 promotes angiogenesis-dependent bone formation and reverses osteoporosis [J]. Nat Commun 11(1):460

4. Wang L, Zhou F, Zhang P, Wang H, Qu Z, Jia P, Yao Z, Shen G, Li G, Zhao G, Li J, Mao Y, Xie Z, Xu W, Xu Y, Xu Y (2017) Human type $\mathrm{H}$ vessels are a sensitive biomarker of bone mass [J]. Cell Death Dis 8(5): 2760

5. Shah B, Rockman CB, Guo Y, Chesner J, Schwartzbard AZ, Weintraub HS, Adelman MA, Riles TS, Berger JS (2014) Diabetes and vascular disease in different arterial territories $[\mathrm{J}]$. Diabetes Care 37(6):1636-1642

6. Ni Z, Youyang Z, Xiangling P et al (2018) Microangiopathy is associated with bone loss in female type 2 diabetes mellitus patients [J]. Diab Vasc Dis Res 15(5):433-441

7. Cui R, Sun SQ, Zhong N, Xu MX, Cai HD, Zhang G, Qu S, Sheng $\mathrm{H}$ (2020) The relationship between atherosclerosis and bone mineral density in patients with type 2 diabetes depends on vascular calcifications and sex [J]. Osteoporos Int 31(6):1135-1143

8. American Diabetes Association (2014) Standards of medical care in diabetes 2014[J]. Diabetes Care 37:S14-S80

9. American Diabetes Association (2019) 2. Classification and diagnosis of diabetes: standards of medical care in diabetes-2019 [J]. Diabetes Care 42(Suppl 1):S13-S28

10. Cosman F, Beur SJD, Leboff MS et al (2014) Clinician's guide to prevention and treatment of osteoporosis. Osteoporos Int 25(10): 2359-2381

11. Bircan Alan B, Aktan A, Alan S et al (2016) Relationship between osteopenic syndrome and severity of coronary artery disease detected with coronary angiography and Gensini score in men [J]. Clin Interv Aging 11:377-382

12. Xu R, Cheng XC, Zhang Y et al (2018) Association of severity of coronary lesions with bone mineral density in postmenopausal women [J]. Arq Bras Cardiol 110(3):211-216

13. Xu R, Yang HN, Li YQ, Wang QF, Guo AH, Ayiti A, Chen XC, Gong R, Banu G, Jian LD, Gao Y, Sheng K, Maimti Y (2016) Association of coronary artery calcium with bone mineral density in postmenopausal women [J]. Coron Artery Dis 27(7):586-591 
14. Tomiyama H, Okazaki R, Koji Y, Usui Y, Hayashi T, Hori S, Yamashina A (2005) Elevated C-reactive protein: a common marker for atherosclerotic cardiovascular risk and subclinical stages of pulmonary dysfunction and osteopenia in a healthy population [J]. Atherosclerosis. 178(1):187-192

15. Papaconstantinou J (2019) The role of signaling pathways of inflammation and oxidative stress in development of senescence and aging phenotypes in Cardiovascular Disease [J]. Cells. 8(11):1383

16. Khosla S (2011) The bone and beyond: a shift in calcium [J]. Nat Med 17(4):430-431

17. Starup-Linde J, Vestergaard P (2016) Biochemical bone turnover markers in diabetes mellitus - a systematic review [J]. Bone. 82: 69-78

18. Wu M, Rementer C, Giachelli CM (2013) Vascular calcification: an update on mechanisms and challenges in treatment $[\mathrm{J}]$. Calcif Tissue Int 93(4):365-373

19. Lehto S, Niskanen L, Suhonen M, Rönnemaa T, Laakso M (1996) Medial artery calcification. A neglected harbinger of cardiovascular complications in non-insulin-dependent diabetes mellitus [J]. Arterioscler Thromb Vasc Biol 16(8):978-983

20. London GM (2003) Arterial media calcification in end-stage renal disease: impact on all-cause and cardiovascular mortality [J]. Nephrol Dial Transplant 18(9):1731-1740

21. Blacher J, Guerin AP, Pannier B, Marchais SJ, London ǴM (2001) Arterial calcifications, arterial stiffness, and cardiovascular risk in end-stage renal disease [J]. Hypertension. 38(4):938-942

22. McCullough PA, Agrawal V, Danielewicz E et al (2008) Accelerated atherosclerotic calcification and Monckeberg's sclerosis: a continuum of advanced vascular pathology in chronic kidney disease [J]. Clin J Am Soc Nephrol 3(6):1585-1598
23. Kang K (2015) Low bone mineral density is associated with intracranial posterior circulation atherosclerosis in women $[\mathrm{J}]$. Bone. 81 : 669-674

24. Jorgensen L, Jenssen T, Ahmed L et al (2007) Albuminuria and risk of nonvertebral fractures [J]. Arch Intern Med 167(13):1379-1385

25. Choi SW, Kim HY, Ahn HR, Lee YH, Kweon SS, Choi JS, Rhee JA, Nam HS, Jeong SK, Park KS, Ryu SY, Song HR, Shin MH (2013) Association of bone mineral density with albuminuria and estimated glomerular filtration rate: the Dong-gu Study [J]. Kidney Blood Press Res 37(2-3):132-141

26. Collins TC, Ewing SK, Diem SJ, Taylor BC, Orwoll ES, Cummings SR, Strotmeyer ES, Ensrud KE, Osteoporotic Fractures in Men (MrOS) Study Group (2009) Peripheral arterial disease is associated with higher rates of hip bone loss and increased fracture risk in older men [J]. Circulation. 119(17):23052312

27. Rencken ML, Chesnut CHI, Drinkwater BL (1996) Bone density at multiple skeletal sites in amenorrheic athletes [J]. JAMA 276(3): 238

28. Chen Y, Guo Q, Zhang M, Song S, Quan T, Zhao T, Li H, Guo L, Jiang T, Wang G (2016) Relationship of serum GDF11 levels with bone mineral density and bone turnover markers in postmenopausal Chinese women [J]. Bone Res 4:16012

29. Yudkin J, Forrest R, Jackson C (1988) Microalbuminuria as predictor of vascular disease in non-diabetic subjects. Islington Diabetes Survey [J]. Lancet. 332(8610):530-533

Publisher's note Springer Nature remains neutral with regard to jurisdictional claims in published maps and institutional affiliations. 